\title{
Expression of $K L F 9$ in pancreatic cancer and its effects on the invasion, migration, apoptosis, cell cycle distribution, and proliferation of pancreatic cancer cell lines
}

\author{
ZHIWEI ZHONG $^{1 *}$, FAN ZHOU $^{1 *}$, DONG WANG ${ }^{1}$, MINGMING WU $^{1}$, WEIMIN ZHOU ${ }^{2}$, \\ YEQING ZOU ${ }^{3}$, JIE $\mathrm{LI}^{3}$, LINQUAN WU ${ }^{1}$ and XIANGBAO YIN ${ }^{1}$ \\ Departments of ${ }^{1}$ Hepatopancreatobiliary Surgery, ${ }^{2}$ Vascular Surgery and ${ }^{3}$ Central Laboratory, \\ The Second Affiliated Hospital of Nanchang University, Nanchang, Jiangxi 330006, P.R. China
}

Received May 7, 2018; Accepted September 26, 2018

DOI: $10.3892 /$ or.2018.6760

\begin{abstract}
Kruppel-like factor 9 (KLF9), a transcription factor, is critical for the inhibition of growth and development of tumors, whereas its effects in pancreatic cancer remains unclear. The purpose of the present study was to investigate the expression and functional significance of KLF9 in vitro, by assessing the expression of KLF9 in pancreatic cancer tissue samples and its association with the total survival of patients and clinicopathological data. The levels of KLF9 expression in adjacent tissues and pancreatic cancer tissues were detected using immunohistochemistry. Using western blot analyses, we assessed KLF9 expression in human pancreatic cancer cell lines. Using flow cytometric analysis and CCK-8, we evaluated the effects of KLF9 expression on cell apoptosis, the cell cycle and proliferation of pancreatic cancer cells. Its effects on migration and cell invasion were detected by performing Transwell assay. By conducting western blot analyses, we evaluated the expression of relative target proteins (involved in invasion, migration, apoptosis, and cell cycle distribution. Our results revealed that in both tissue samples and cell lines (particularly in BxPC-3 and PANC-1 cells) of pancreatic cancer, KLF9 exhibited relatively lower expression. In addition, low KLF9 expression was related to the differentiation $(\mathrm{P}<0.001)$ and depth of vascular invasion $(\mathrm{P}=0.016)$ and was associated with a poor overall survival rate. In PANC-1 and BxPC-3 cells,
\end{abstract}

Correspondence to: Professor Xiangbao Yin, Department of Hepatopancreatobiliary Surgery, The Second Affiliated Hospital of Nanchang University, 1 Minde Road, Nanchang, Jiangxi 330006, P.R. China

E-mail: yxb115@sina.com

${ }^{*}$ Contributed equally

Abbreviations: KLF9, Kruppel-like transcription factor 9; EMT, epithelial-mesenchymal transition; CCK-8, Cell Counting Kit-8

Key words: pancreatic cancer, KLF9, prognosis, cell cycle arrest, apoptosis, EMT
KLF9 overexpression decreased the proliferation of pancreatic cancer cells, induced apoptosis, blocked the cell cycle at the $\mathrm{S}$ phase, and inhibited the migration and invasion of tumor cells. KLF9 overexpression downregulated MMP-9, MMP-2 $\mathrm{Bcl}-2, \mathrm{~N}$-cadherin and cyclin $\mathrm{B}$, and upregulated the levels of E-cadherin, Bax, p53, CDK4 and cyclin D1. On the whole, our findings indicated that KLF9 exhibited low expression in pancreatic cancer, and upregulation of KLF9 may inhibit the progression of pancreatic cancer. KLF9 may have potential diagnostic and therapeutic values in this type of cancer.

\section{Introduction}

Pancreatic cancer is malignancy of the digestive system. The overall survival outlook for pancreatic cancer worldwide is still grim. Although the survival rate of pancreatic cancer patients has steadily increased, progress in research is very slow and there is still no treatment that is effective and no early diagnosis that is reliable (1-3). Pancreatic cancer remains the most severe cancer worldwide, with a total survival rate in 5 years below $5 \%$ (4). Thus, searching for more effective treatments and strategies to improve the therapeutic effect, and discovering the generation and molecular mechanisms of metastasis in pancreatic cancer is urgently needed. The family of Krupple-like transcription factor (KLF) is comprised of 17 distinctive members, which have a variety of cellular functions (5). KLF9 is one of the most important genes. KLF9 is an important nuclear transcription factor expressed widely and conserved highly in higher mammals and human tissues such as the brain, liver and uterus (6,7). KLF9 is a member of the KLF transcription factor family (8). Studies in tumors have revealed that the role of KLFs is conditionally dependent (9), and possess tumor suppressive and proto-oncogenic effects on tumors $(10,11)$. A recent study (12) revealed that KLF9 exhibited low expression in the esophagus and could bind to TCF4 to inhibit the $\beta$-catenin/TCF signaling pathway, thus suppressing tumor growth. In colorectal cancer, compared with normal tissue, KLF9 transcription and protein levels were revealed to be significantly downregulated (13). In endometrial cancer, KLF9 expression was also significantly downregulated. In these tumors, inhibition of downstream molecules of actin 
skeletal protein regulatory factors was detected after overexpression of KLF9 (14). However, the expression of KLF9 in pancreatic cancer has not been investigated. In addition, the effects of KLF9 on apoptosis, the cell cycle and epithelialmesenchymal transition (EMT) in pancreatic cancer remain to be fully elucidated.

More and more evidence has revealed that KLF9 is not only involved in many important biological events, including cell proliferation growth (15) and immune response (16), but is also closely related to the occurrence and development of multiple tumors (17-20). We observed that upregulation of KLF9 expression had an obvious effect on proliferation, apoptosis, invasion and migration of PANC-1 and BxPC-3 cells in vitro and this study may provide data on KLF9 as a prognostic marker and therapeutic target drug on pancreatic cancer.

\section{Materials and methods}

Patients and tissue samples. The Second Affiliated Hospital of Nanchang University provided 60 pairs of specimens of pancreatic cancer and matched paracancerous tissue. Using histopathology according to the World Health Organization (WHO) standards, these patients (32 male and 28 female patients; 26 patients under the age of 60 and 34 patients over 60 years) were diagnosed with pancreatic cancer (21) and underwent surgery from January 2011 to March 2015. Fresh tissue samples were cut into 4-mm cubes, and snap-frozen in liquid nitrogen and stored at $-80^{\circ} \mathrm{C}$. According to the American Cancer Joint Committee (AJCC), clinical data, including distant metastasis, lymph node metastasis, depth of invasion, tumor differentiation, tumor location, tumor size, age and sex, were obtained from medical records (22). The Ethics Committee of the Second Affiliated Hospital of Nanchang University approved our study, and all participants signed and provided written informed consent. This study was conducted by the ethical standards of the Helsinki Declaration.

Immunohistochemistry. Paraffin-embedded tissue were cut into $4-\mu \mathrm{m}$ thick sections and stained with hematoxylin and eosin. Using a two-step immunohistochemistry method, we first deparaffinized the tissue sections two times in xylene, 10 min each time, rehydrated with a fractional alcohol series (100-50\%) for $2 \mathrm{~min}$, and then the sections underwent antigen repair at $130^{\circ} \mathrm{C}$ for $10 \mathrm{~min}$ in a pressure cooker. To block the potential endogenous peroxidase activity, we cultured the sections with hydrogen peroxide $(0.3 \%)$, washed them 3 times with phosphate-buffered saline (PBS) for $3 \mathrm{~min}$, and then placed them at room temperature with $20 \%$ normal goat serum for $30 \mathrm{~min}$. Then, we cultured the sections with mouse monoclonal anti-KLF9 antibody (cat. no. sc-517075; Santa Cruz Biotechnology, Inc., Dallas, TX, USA) at a dilution of 1:200 at $4^{\circ} \mathrm{C}$ overnight. The following day it was washed three times with PBS. Next, the sections were cultured with biotin-labeled goat anti-mouse IgG (dilution 1:200; cat. no. PV-6000ZSGB-Bio; OriGene Technologies, Inc., Beijing, China) at $37^{\circ} \mathrm{C}$ for $30 \mathrm{~min}$. The slices were reacted with 3,3'-diaminobenzidine solution, stained with hematoxylin and covered with coverslips after being washed with PBS three times. Immunohistochemistry sections were then examined and scored independently using blind pathology on a light microscope (Nikon Corp., Tokyo, Japan). The scoring system was calculated according to the dyeing intensity (grade 0 indicated no staining; grade 1 indicated mild staining; grade 2 indicated moderate staining; grade 3 indicated strong staining) and the percentage of dyeing (grade 0 indicated no staining; grade 1 indicated staining percentage $<10 \%$; grade 2 indicated dyeing percentage $10-40 \%$; grade 3 indicated dyeing percentage $>40 \%$ ). Then, according to previous studies, the total staining index was acquired by multiplying each immune staining score by 0 to 9 , as follows: $0-1$ indicated KLF9 negative expression, or 2-9 indicated KLF9 positive expression (23).

Culture of cell lines. The Shanghai Institute of Biochemistry and Cell Biology (Shanghai, China) provided Sw1990, BxPC-3, Capan-1 and PANC-1 pancreatic cancer cell lines. Rui-Lu Biotech (Shanghai, China) provided the non-transformed pancreatic epithelial cell line HPDE6c7 (non-cancerous line). We cultured cells in high glucose medium DMEM (DMEM; Invitrogen; Thermo Fisher Scientific, Inc., Waltham, MA, USA) with 9 and $10 \%$ fetal bovine serum (FBS; Hyclone; GE Healthcare Life Sciences, Logan, UT, USA) and 1\% sodium pyruvate (Invitrogen; Thermo Fisher Scientific, Inc.) in a humidified incubator with $95 \%$ air and $5 \% \mathrm{CO}_{2}$ maintained at $37^{\circ} \mathrm{C}$. We grew cells in the exponential phase and sub-cultured them at nearly $80 \%$ confluence.

Protein extraction and immunoblotting. Using RIPA buffer (Nanjing KeyGen Biotech Co., Ltd., Nanjing, China) with $1 \%$ phenylmethanesulfonyl fluoride, we extracted total cell proteins. We quantified them with the Bradford method. Next, we separated the protein samples ( $20 \mu \mathrm{g}$ per lane) in SDS-PAGE capsules with $10 \%$ sodium dodecyl sulfate-polyacrylamide gel electrophoresis (SDS-PAGE). We then transferred them to polyvinylidene difluoride membranes (EMD Millipore, Billerica, MA, USA). In the immunoblotting process, we first cultured the membranes with $5 \%$ bovine serum albumin (BSA) in Tris-buffered saline-Tween-20 (TBS-T). Next, we cultured them overnight at $4^{\circ} \mathrm{C}$ with the corresponding primary antibody. The following day, we washed the membranes three times with TBS-T, each time for $10 \mathrm{~min}$. Then, the membranes were cultured with horseradish peroxidase conjugated secondary antibody for $1 \mathrm{~h}$ at ambient temperature followed by enhanced chemiluminescent reagent (ECL; EMD Millipore). The proteins CDK4 (cat. no. PKA-325), cyclin B (cat. no. 7518) and cyclin D1 (cat. no. P4190-10) were obtained from bioWORLD (Dublin, OH, USA); p53 (cat. no. 2527), MMP-9 (cat. no. 13667), MMP-2 (cat. no. 13132), N-cadherin (cat. no. 13116), E-cadherin (cat. no. 3195), Bcl-2 (cat. no. 4223) and Bax (cat. no. 2772) were purchased from Cell Signaling Technology, Inc. (Danvers, MA, USA). We applied all the antibodies following the instructions of the manufacturers. Anti-GAPDH (ZSGB-Bio; OriGene Technologies, Inc.) served as an endogenous reference. All the primary antibodies were diluted at a ratio of 1:1,000 and the secondary antibody was diluted at a ratio of 1:5,000.

saRNA and gene transfection. We obtained Human KLF9 saRNA reagent from Shanghai GenePharma Co., Ltd. (Shanghai, China), with the sequences: KLF9_s1, 
5'-UGUGCAGUAAUCCUUCCAGTT-3', KLF9_s2, 5'-UUCGAUCGCUUGAUCAUGCTT-3' and KLF9_s3, 5'-UUAACGUGAUUCAAGAGAGTT-3' respectively. GAPDH saRNA served as the positive control sequence (5'-UGACCUCAACUACAUGGUUTT-3') and the negative control sequence was 5'-UUCUCCGAACGUGUCACGUTT-3'. The pancreatic cancer BxPC-3 and PANC-1 cell lines grew in a six-well plate with 95\% air and 5\% CO2 using Lipofectamine 2000 (Invitrogen; Thermo Fisher Scientific, Inc.) reagent following the manufacturer's instructions on transient transfection of saRNAs. After $48 \mathrm{~h}$, the transfected cells could be used for further assay or protein extraction.

Flow cytometric cell cycle and apoptosis assays. An saRNA fragment was transfected transiently by using Lipofectamine 2000 and the cell cycle distribution was analyzed as follows $48 \mathrm{~h}$ later. Cells $\left(1 \times 10^{6}\right)$ were harvested with trypsin digestion, washed using PBS, and fixed using 70\% ethanol overnight at $4^{\circ} \mathrm{C}$. The following day, we re-suspended the cells in $500 \mu \mathrm{l}$ of propidium iodide (PI)/RNase staining solution (Sungene Biotech Co., Ltd., Tianjin, China) and cultured for $30 \mathrm{~min}$ at $37^{\circ} \mathrm{C}$. Next, we studied the samples using a FACScan flow cytometer (BD Immunocytometry Systems, San Jose, CA, USA). For the evaluation of cell apoptosis, using the Annexin V-FITC/PI Apoptosis Detection kit (Nanjing KeyGen Biotech Co., Ltd.), we assessed the cells duplicated from the cell cycle analysis, following the manufacturer's instructions. In brief, we washed these cells in ice-cold PBS and cultured them with Annexin V-FITC and PI solutions for $15 \mathrm{~min}$ in the dark. Next, using FACScan flow cytometry, we studied them for apoptosis. We studied no less than 105 cells for each sample, which were analyzed by flow cytometry (BD Biosciences, San Jose, CA, USA) within $1 \mathrm{~h}$. Finally, we summarized the data as the mean \pm standard error (SD).

Cell proliferation assay. Using CCK-8 assay kit (KeyGen Biotech Co., Ltd.) after cell KLF9 overexpression, we assessed cell proliferation. We seeded the cells in 96-well plates at a density of 5,000 cells/well, cultured them for $12 \mathrm{~h}$, and then promptly transfected them using the saRNAs aforementioned. The cells were cultured for 24, 48, 72 and $96 \mathrm{~h}$, respectively. At the end of each experiment, $10 \mu \mathrm{l}$ of the CCK-8 reagent cell culture medium was added to each well; then the mixture was cultured for another hour and assessed under a microplate reader at an optical density of $450 \mathrm{~nm}$ (PerkinElmer, Inc., Waltham, MA, USA). The data was expressed as the mean \pm standard error. Each group was set up with three wells and repeated at least 3 times.

Transwell migration and invasion of tumors. Migration and invasion of tumor cells were assessed using a modified 24-well filter Boyden chamber with the filter either precoated or uncoated with Matrigel (BD Biosciences, Shanghai, China). At a concentration of $1 \times 10^{6}$ cells $/ \mathrm{ml}$, saRNA cells grew and were transfected transiently with pancreatic cancer cells in serum-free DMEM. Next, $200 \mu 1$ of cell solution was added into the upper chamber of a filter with $8-\mu \mathrm{m}$ pores and DMEM with $20 \%$ FBS was added in the bottom chamber. After being cultured for $48 \mathrm{~h}$, the non-invaded cells and
Table I. Relationship between the expression level of KLF9 and the clinicopathological parameters of pancreatic cancer patients.

\begin{tabular}{|c|c|c|c|c|}
\hline \multirow[b]{2}{*}{$\begin{array}{l}\text { Clinical } \\
\text { parameters }\end{array}$} & \multirow[b]{2}{*}{$\mathrm{n}$} & \multicolumn{2}{|c|}{ KLF9 expression } & \multirow[b]{2}{*}{ P-value } \\
\hline & & $\begin{array}{l}\text { High } \\
{[\mathrm{n}(\%)]}\end{array}$ & $\begin{array}{c}\text { Low } \\
{[\mathrm{n}(\%)]}\end{array}$ & \\
\hline Sex & & & & 0.712 \\
\hline Male & 32 & $5(15.6)$ & $27(84.4)$ & \\
\hline Female & 28 & $3(10.7)$ & $25(89.3)$ & \\
\hline Age (years) & & & & 0.717 \\
\hline$<60$ & 26 & $4(15.4)$ & $22(84.6)$ & \\
\hline$\geq 60$ & 34 & $4(11.8)$ & $30(88.2)$ & \\
\hline Tumor location & & & & 0.067 \\
\hline Head and neck & 34 & $2(5.9)$ & $32(94.1)$ & \\
\hline Body and tail & 26 & $6(23.1)$ & $20(76.9)$ & \\
\hline Tumor differentiation & & & & $<0.001$ \\
\hline High & 5 & $5(100)$ & $0(0)$ & \\
\hline Medium & 18 & $3(16.7)$ & $15(83.3)$ & \\
\hline Low & 37 & $0(15)$ & $37(100)$ & \\
\hline AJCC stage & & & & 0.698 \\
\hline I-II & 37 & $6(16.2)$ & $31(83.8)$ & \\
\hline III-IV & 23 & $2(8.7)$ & $21(91.3)$ & \\
\hline Nerve invasion & & & & 0.429 \\
\hline No & 16 & $3(18.8)$ & $13(81.2)$ & \\
\hline Yes & 44 & $5(11.4)$ & $39(88.6)$ & \\
\hline Vascular invasion & & & & 0.016 \\
\hline No & 35 & $8(22.9)$ & $27(77.1)$ & \\
\hline Yes & 25 & $0(0)$ & $25(100)$ & \\
\hline Lymph node metastasis & & & & 0.130 \\
\hline Absent (N0) & 32 & $2(6.3)$ & $30(93.7)$ & \\
\hline Present (N1-3) & 28 & $6(21.4)$ & $22(78.6)$ & \\
\hline
\end{tabular}

All data are expressed as the number of patients (\%). P-values were calculated using SPSS 17.0 using a Chi-square test. P-values $<0.05$ were considered to indicate statistical significance. KLF9, Kruppel-like factor 9; AJCC stage, American cancer Joint Committee stage.

Matrigel were wiped away carefully. Cells that had migrated or invaded the lower chamber of the filter were fixed in $4 \%$ paraformaldehyde and stained with $0.5 \%$ crystal violet. At an x10 magnification under inverted microscope, we counted the number of invaded or migrated cells in 5 randomly selected fields (Nikon Corp.).

Statistical analysis. Using the Kaplan-Meier curve, we assessed total survival and KLF9 expression was assessed using the $\log$ rank test. The in vitro data are expressed as the means \pm standard error and performed using one-way analysis of variance. Multiple comparison between the groups was performed using a Student-Newman-Keuls (S-N-K) test. All assays were performed independently three times. Using SPSS v17.0 software (SPSS, Inc., Chicago, IL, USA), we conducted all statistical analyses. A value of $\mathrm{P}<0.05$ was considered to indicate a statistically significant difference. 
A

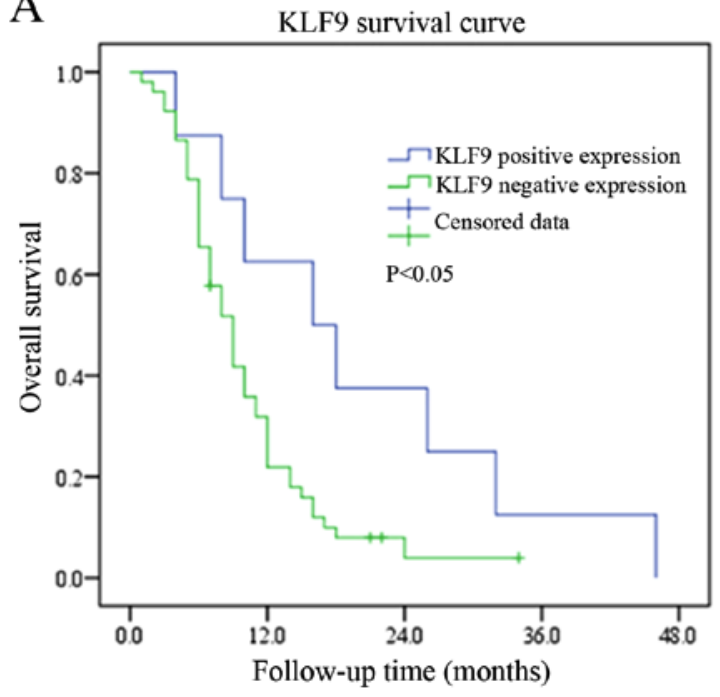

B
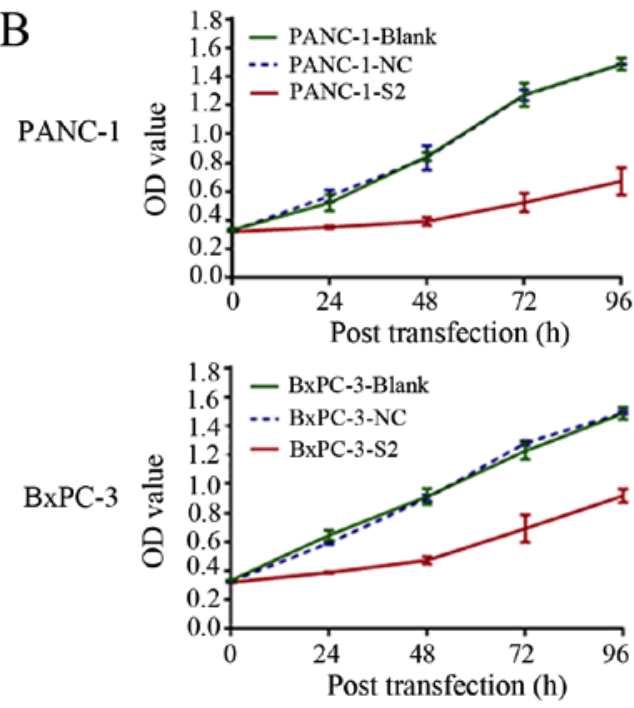

Figure 1. (A) Low expression of KLF9 in pancreatic cancer and its association with survival in patients with pancreatic cancer. Kaplan-Meier curves stratified by the expression level of KLF9 in pancreatic cancer which revealed a significant association between the expression level of KLF9 and the overall survival rates. (B) KLF9-S2 was transiently transfected into pancreatic cancer cells, and then CCK- 8 cell viability determination was performed. The effects of KLF9 saRNAs on PANC-1 and BxPC-3 cell growth over 96 h. (NC vs. S2, P<0.01). KLF9, Kruppel-like factor 9; saRNA, small activating RNA; S2, saRNA 2; NC, negative control.

A

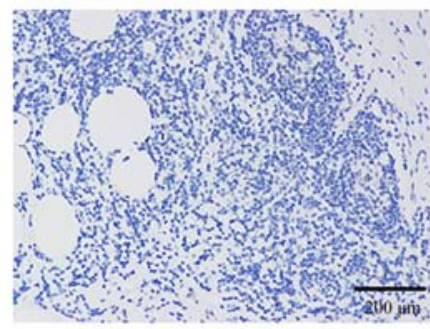

C

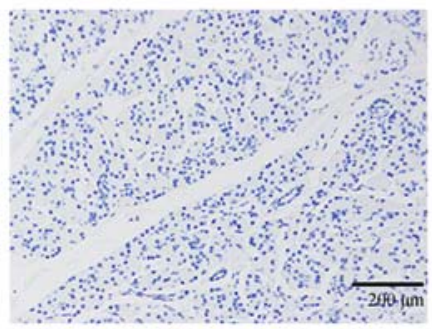

E

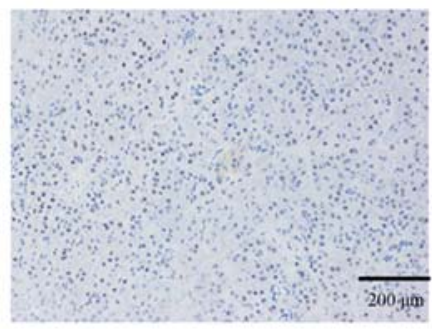

G

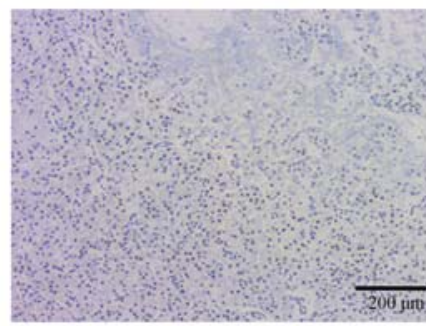

B

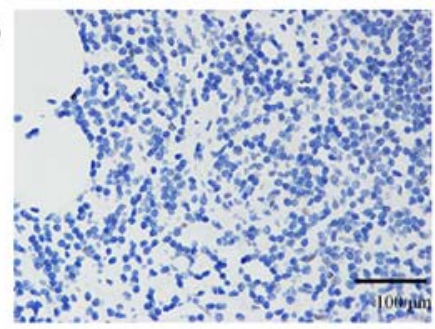

D

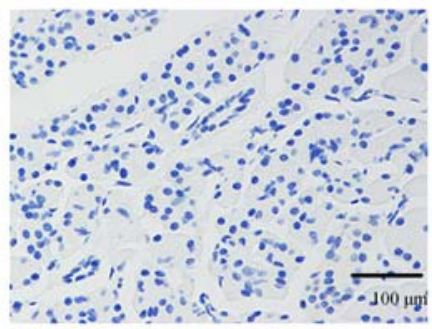

$\mathrm{F}$

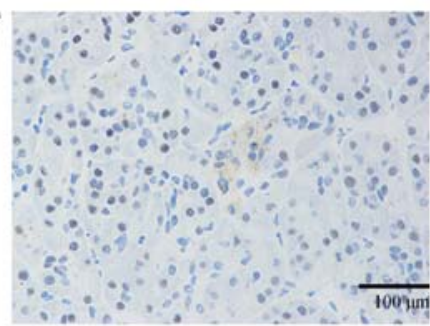

$\mathrm{H}$

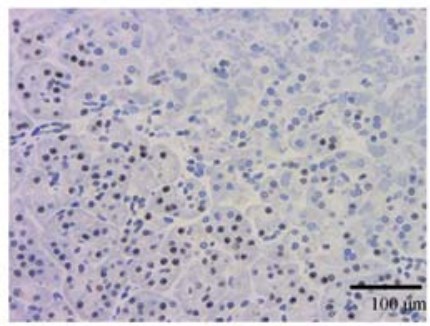

Figure 2. Expression of KLF9 in pancreatic cancer tissues and paired normal tissues. Immunohistochemical staining of KLF9 protein in normal pancreatic tissues. KLF9 protein was localized in the pancreatic cell nuclei. (A and B) KLF9 protein was negative in pancreatic cancer tissues. (C and D) Negative KLF9 staining in paired normal tissues. (E and F) Weak positive KLF9 staining in paired normal tissues. (G and H) Strong positive KLF9 staining in paired normal tissues. KLF9, Kruppel-like factor 9. 

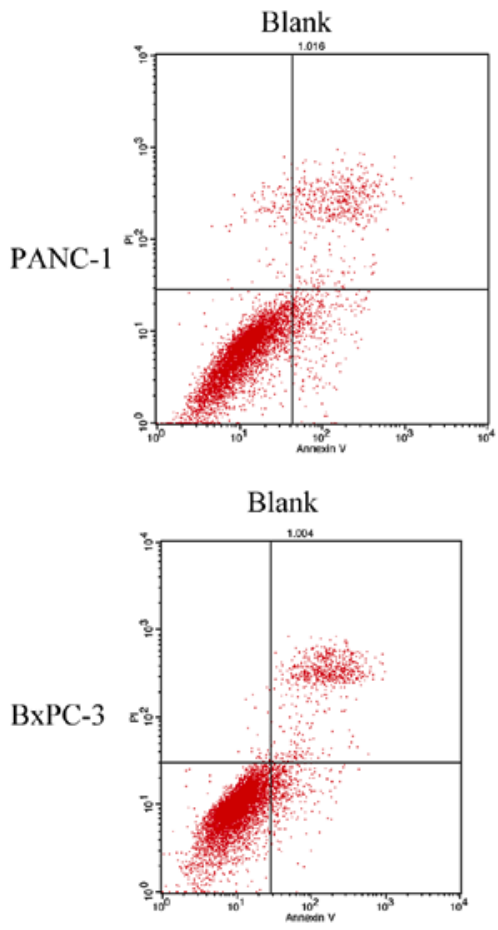
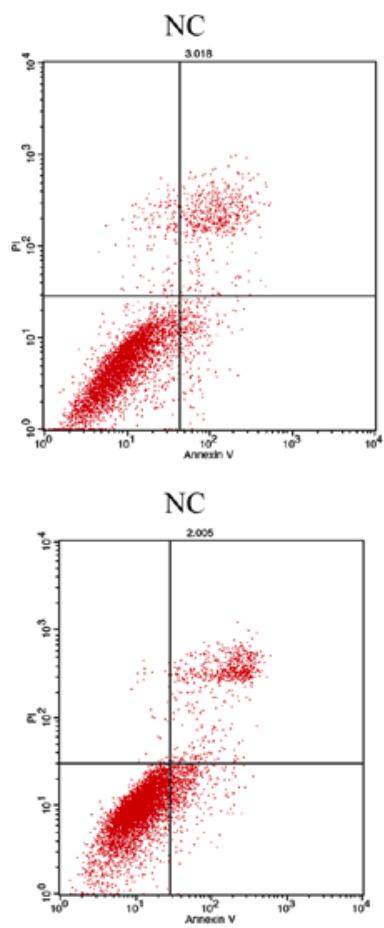
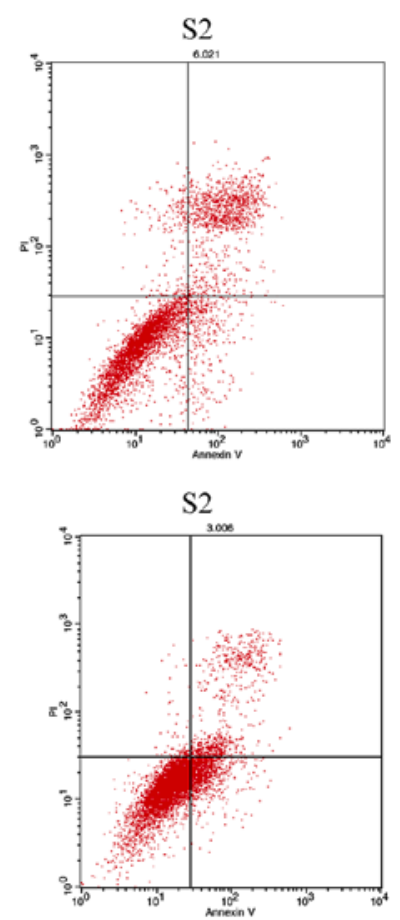

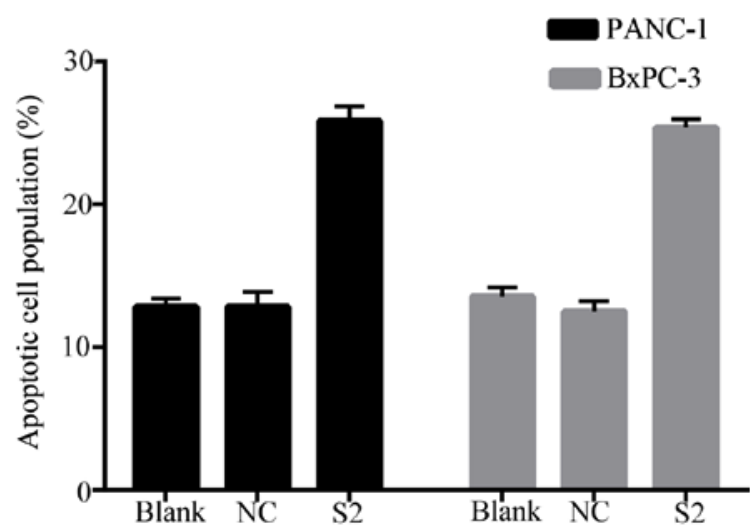

Figure 3. Induction of apoptosis by saRNA. Fluorescence-activated cell sorting assessment of KLF9 saRNA induction of apoptosis in PANC-1 and BxPC-3 cells. (NC vs. S2, P<0.01). KLF9, Kruppel-like factor 9; saRNA, small activating RNA; S2, saRNA 2; NC, negative control; PI, propidium iodide.

\section{Results}

The relationship between the clinicopathological features of pancreatic cancer and the expression of KLF9 was revealed in Table I. Our data indicated that the expression of KLF9 in tumor tissue was related to the depth of vascular invasion $(\mathrm{P}=0.016)$ and differentiation $(\mathrm{P}<0.001)$. However, KLF9 expression was not related to tumor location, age, sex, metastasis of lymph node, nerve invasion and TNM stage. The Kaplan-Meier curve was used to analyze the relationship between the expression of KLF9 in pancreatic cancer and the total survival of patients. Our data revealed that overexpression of KLF9 was related to the total survival of these patients (P<0.05; Fig. 1A).

We evaluated the effect of KLF9 overexpression on the proliferation, cell cycle distribution, and apoptosis of pancreatic cancer cells. In particular, KLF9 overexpression significantly reduced the proliferation abilities of PANC-1 and BxPC-3 cells in comparison with positive saRNA control cells $(\mathrm{P}<0.01 ;$ Fig. 1B). The expression of KLF9 in pancreatic cancer and matched paracancerous tissue samples was first evaluated here, and KLF9 revealed low expression or no expression $(52 / 60,86.67 \%)$ in pancreatic cancer tissues, whereas it exhibited a high expression in adjacent tissues of pancreatic cancer $(56 / 60,93.33 \%$ ) (Fig. 2). Thus, compared with the expression in matched paracancerous tissues the expression of KLF9 in 52 pancreatic cancer tissues was lower $(\mathrm{P}<0.001)$.

Furthermore, we found that transfection of KLF9 saRNA into BxPC-3 and PANC-1 cells significantly increased the number of apoptotic $(\mathrm{P}<0.01 ;$ Fig. 3$)$ cells. Cell cycle profile data revealed that overexpression of KLF9 significantly increased the number of cells in the $\mathrm{S}$ phase from $<30 \%$ to $>40 \%$ ( $\mathrm{P}<0.01$; Fig. 4).

Since our in vitro data revealed that low expression of KLF9 was related to metastasis of pancreatic cancer, we assessed whether KLF9 overexpression inhibited the invasion and migration of pancreatic cancer cells. It was revealed by the data that KLF9 saRNA significantly decreased the migration and invasive abilities of BxPC- 3 and PANC- 1 cells $(\mathrm{P}<0.05$; Fig. 5). 

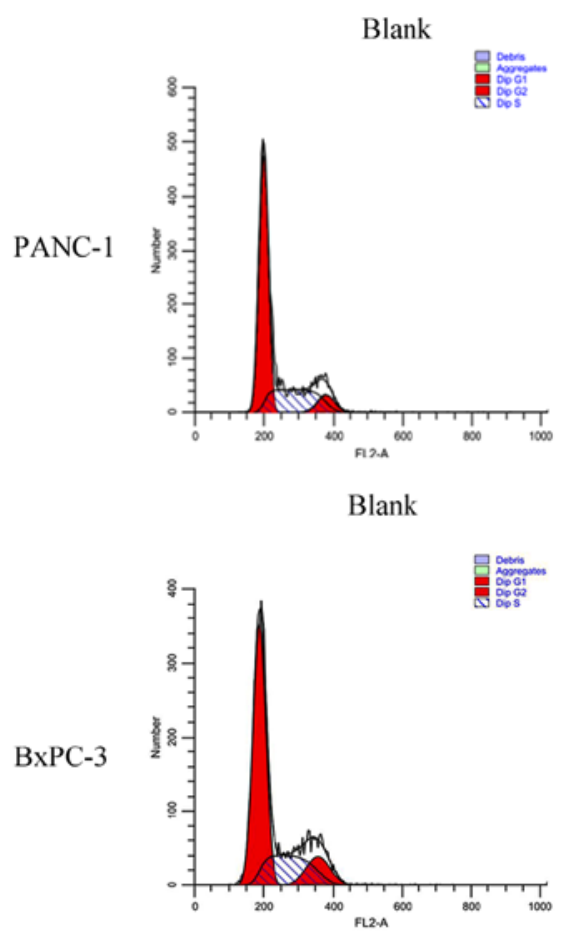

PANC-

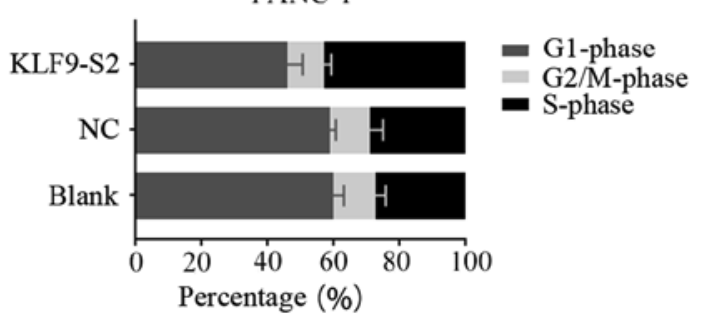

NC
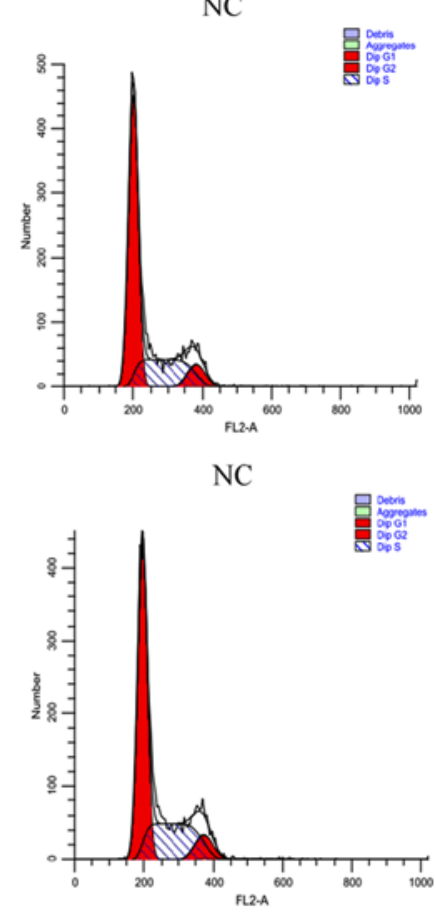

KLF9-S2

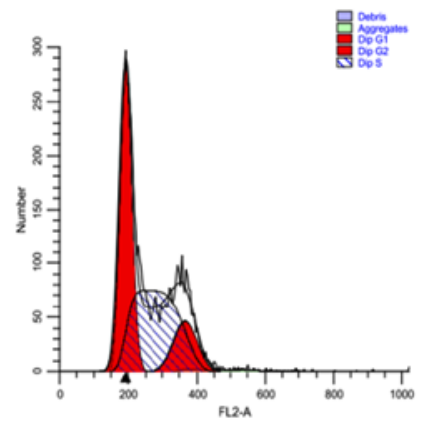

KLF9-S2

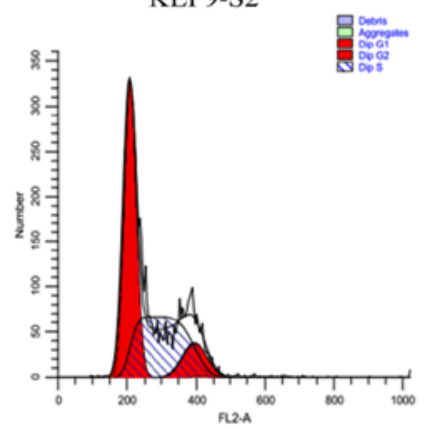

BxPC-3

Figure 4. Cell cycle distribution. KLF9 overexpression was performed to detect cell cycle distribution in PANC-1 and BxPC-3 cells. KLF9-S2 was transiently transfected into PANC-1 and BxPC-3 cells, and then the cells were subjected to flow cytometry to analyze the cell cycle. (NC vs. S2, P<0.01). KLF9, Kruppellike factor 9; saRNA, small activating RNA; S2, saRNA 2; NC, negative control.

The potential molecular events in KLF9 overexpression in pancreatic cancer cells was further explored. The data indicated that KLF9 regulated the cell cycle progression of pancreatic cancer cells. In addition, we found that overexpression of KLF9 increased the expression of $\mathrm{p} 53$ and Bax and downregulated Bcl-2 in PANC-1 and BxPC-3 cells (Fig. 6A). The data further indicated that KLF9 overexpression significantly upregulated the levels of proteins cyclin D1 and CDK4, and downregulated cyclin B (Fig. 6B). Next, tumor cell EMT-related gene expression was assessed. In the course of cell EMT, epithelial marker expression of E-cadherin was increased, yet cell mobility markers, MMP-9 and MMP-2, and mesenchymal marker expression $\mathrm{N}$-cadherin were reduced. The data revealed that KLF9 overexpression could significantly modulate protein expression (Fig. 6C).

KLF9 protein expression in PC cell lines (Sw1990, BxPC-3, Capan-1, and PANC-1) was significantly lower than that in the non-transformed pancreatic epithelial cell line HPDE6c7 (Fig. 6D). This indicated an association between low expression of KLF9 and pancreatic cancer. To demonstrate the role of KLF9 in the development and progression of pancreatic cancer, we used a KLF9 saRNA construct to promote the expression of KLF9 in PANC-1 and BxPC-3 cell lines. We found that KLF9 was significantly overexpressed after $48 \mathrm{~h}$ of saRNA transfection and KLF9-S2 was screened from the three interference fragments (KLF9-S1, KLF9-S2, and KLF9-S3) (Fig. 6D).

\section{Discussion}

Pancreatic cancer refers to a type of malignancy of the digestive system, whose prognosis is very poor (24). Despite progress in immunotherapy, radiotherapy, chemotherapy and surgery in recent decades, effective treatment of pancreatic cancer still poses a main clinical challenge. Thus, a new treatment program for pancreatic cancer is required. However, the precise development and occurrence mechanisms of pancreatic cancer are far from fully elucidated. It is still the most aggressive cancer in the world, and the total 5-year survival rate is $<5 \%$. Accordingly, it is urgent to further explore the molecular mechanisms of pancreatic cancer and develop more effective therapeutic strategies to improve the treatment effects. The molecular markers in this study may be helpful for the early diagnosis of pancreatic cancer, which can significantly improve the survival of patients. 

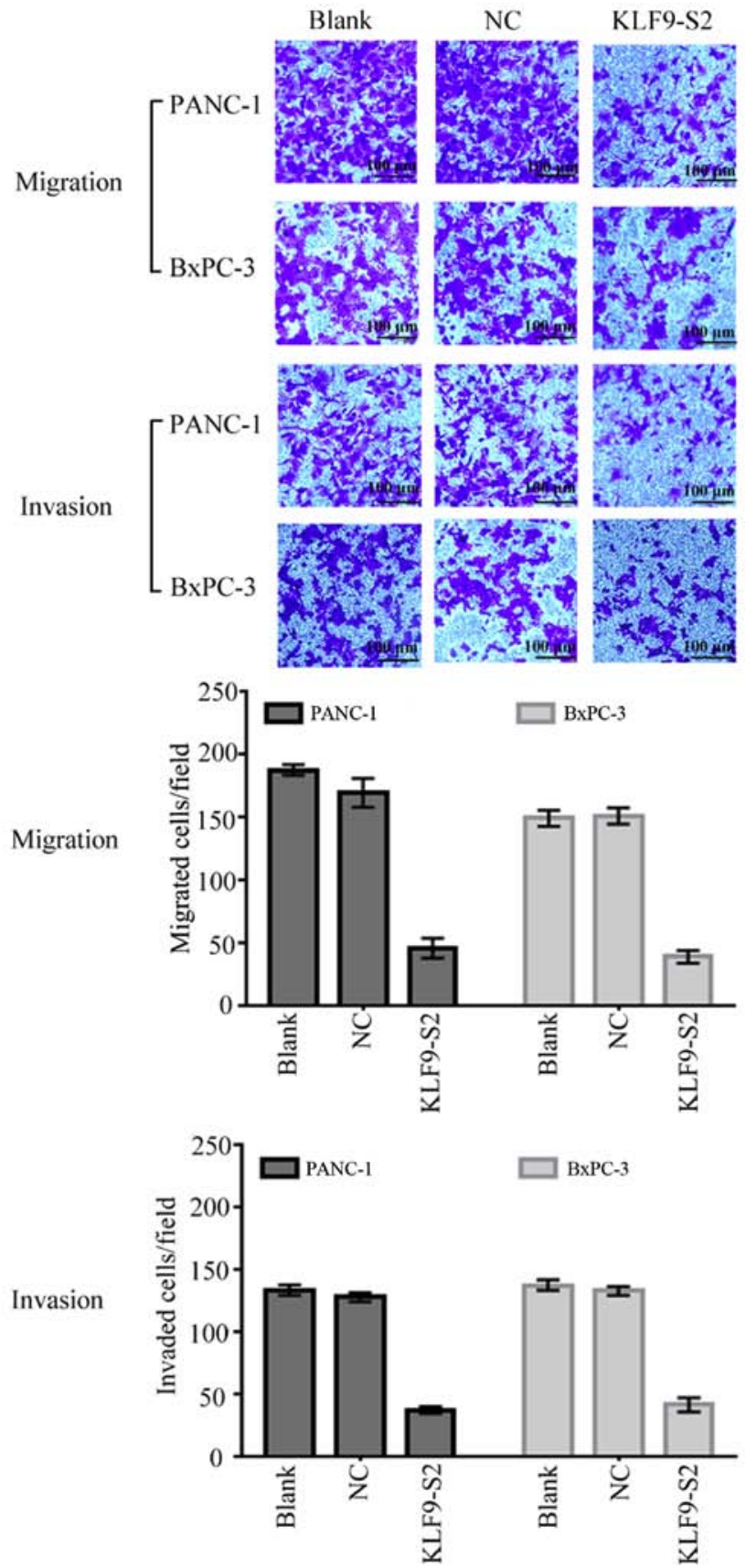

Figure 5. Effect of KLF9 on cell migration and invasion. Overexpression of KLF9 inhibited PANC-1 and BxPC-3 cell invasion and migration. KLF9-S2 was transiently transfected into pancreatic cancer PANC-1 and BxPC-3 cel lines for Transwell migration assays. (NC vs. S2, P<0.05). KLF9, Kruppellike factor 9; saRNA, small activating RNA; S2, saRNA 2; NC, negative control.

In the present study, we found that the expression of KLF9 in pancreatic tumors was related to pancreatic cancer behaviors and the total survival of patients. We then evaluated the effect of the low expression of KLF9 on the malignant behavior of pancreatic cancer cells in vitro. We also determined that KLF9 exhibited low expression in tumor tissue in comparison with adjacent paracancerous tissue, which is relevant to tumor differentiation and vascular invasion. Furthermore, our data revealed that low expression of KLF9 was associated with a poor total survival rate, which is an independent prognostic indicator for pancreatic cancer.
Our data in vitro demonstrated that overexpression of KLF9 could reduce the viability of pancreatic cancer cells and induce arrest of tumor cell apoptosis at the $\mathrm{S}$ phase of the cell cycle. Overexpression of KLF9 could also inhibit migration and invasion capacities of tumor cells in vitro. At the gene level, overexpression of KLF9 upregulated the expression of cyclin D1, CDK4, p53, Bax and E-cadherin, while it downregulated the levels of cyclin B, N-cadherin, MMP-2 and MMP-9. Our study revealed that KLF9 may be used as a prognostic indicator and therapeutic target for pancreatic cancer. In fact, cancer growth is characterized by uncontrolled cell proliferation and transformation. In addition, at the molecular level, numerous genes including proteins that regulate cell proliferation and death as well as genomic stability, are altered $(25,26)$.

In the present study, the human KLF9 gene is located on chromosome $9 \mathrm{q} 13$ and its coding sequence. The coding region is as long as $735 \mathrm{bp}$ and contains two exons that encode a polypeptide chain containing 144 amino acid residues. KLF9 peptide chain amino terminal $~ 84-116$ is rich in Asp/Glu acidic amino acid residues, the carboxyl terminal (143 to 167,173 to 197 and amino acids 203 to 225) is a conserved DNA binding domain containing three tandem/adjacent Cys2/His2 zinc fingers (27). Studies have shown that the DNA copy number of chromosome $9 q$ is reduced in various cancers, which is associated with low expression of $\operatorname{KLF} 9(13,28)$. Though our study did not assess changes in DNA copy number, our data revealed the expression of KLF9 in pancreatic cancer tissues, for the first time.

The low expression of KLF9 was associated with poor progression and total survival in patients with pancreatic cancer, which was consistent with previous studies in cancers of other organs (29,30). Pancreatic cancer is a heterogeneous and complex disease since the development of pancreatic cancer involves a variety of genetic changes (31). Our research focused on the overexpression of KLF9 which inhibited the malignant behavior of pancreatic cancer in vitro, consistent with previous published studies $(17,32)$. For example, when KLF9 was upregulated, the proliferation of pancreatic cells was significantly reduced. As a mitotic modulator, KLF9 can regulate the formation of mitotic spindles and cell mitosis. Tumor cells cannot form a normal level of mitotic spindles and stagnate at the $\mathrm{S}$ phase of the cell cycle. At the molecular level, overexpression of KLF9 can regulate cell proliferation, apoptosis, and expression of multiple genes of EMT, which play an important role in invasion and metastasis of tumor cells. During EMT, tumor cells lose more polarity and obtain the ability to migrate and invade by degradation of the extracellular matrix (ECM) (33). MMP is a family of zincdependent endopeptidases whose function is the reduction of the ECM composition in tissues $(34,35)$. Our data revealed that overexpression of KLF9 inhibited migration and invasion of pancreatic cancer cells by downregulating the expression of MMP-2 and MMP-9. We also found that KLF9 overexpression upregulated the level of E-cadherin and downregulated that of $\mathrm{N}$-cadherin.

A recent study revealed that KLF9 was downregulated in esophageal cancer and could be combined with TCF4 to inhibit beta-catenin/TCF signaling pathways, thus stimulating tumor suppression (12). In addition, KLF9 is considered to be a tumor suppressor gene in many tumors and targeting KLF9 
A

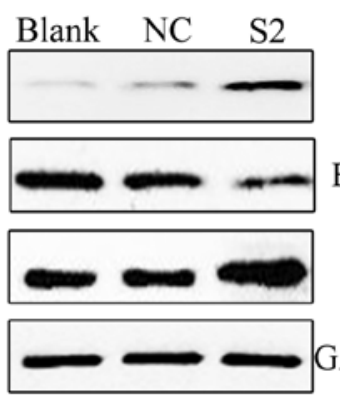

PANC-1

$\mathrm{C}$

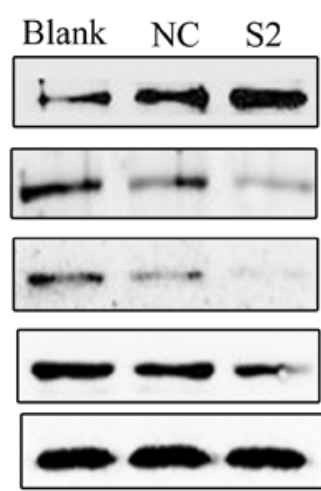

PANC-1

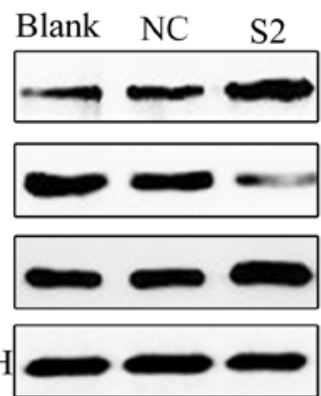

BxPC-3
B

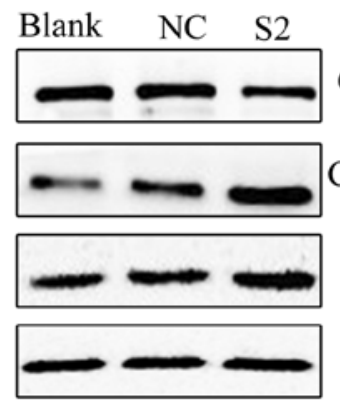

PANC-1

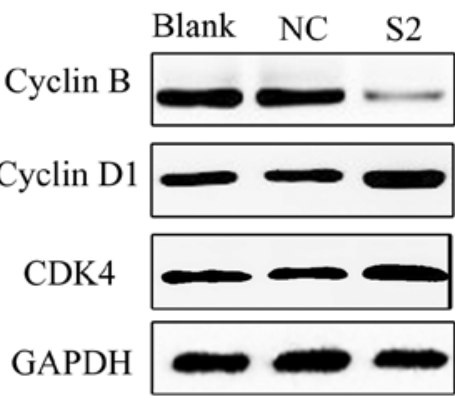

BxPC-3
D HPDE6c7 Sw1990 Capan-1 BxPC-3 PANC-1

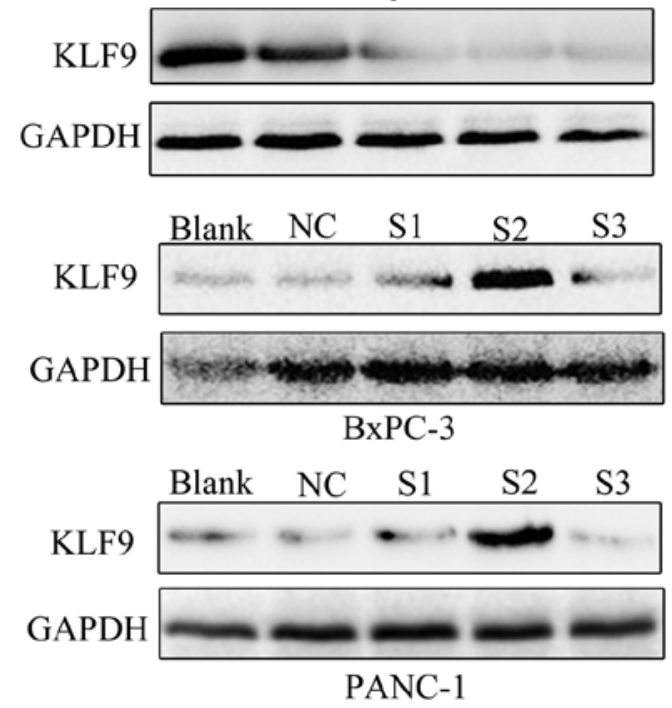

Figure 6. Western blotting. (A) The expression of apoptosis-related proteins. Western blotting revealed downregulated Bcl-2, and upregulated Bax and p53 following overexpression of KLF9 in PANC-1 and BxPC-3 cells. (B) The expression of cell cycle distribution proteins. Western blotting revealed downregulated cyclin B, and upregulated cyclin D1 and CDK4 following overexpression of KLF9 in PANC-1 and BxPC-3 cells. (C) The effect of KLF9 on EMT. Overexpression of KLF9 markedly suppressed N-cadherin, MMP-2 and MMP-9, while it increased E-cadherin expression in PANC-1 and BxPC-3 cells. (D) Western blot analysis of KLF9 protein in pancreatic cancer cell lines and screening of optimal saRNA from KLF9-S1, KLF9-S2 and KLF9-S3. KLF9 was expressed at high levels in HPDE6c7 cells, however in Sw1990 cells it was expressed at a lower level when compared to HPDE6c7, and was almost absent in Capan-1, PANC-1 and BxPC-3 cells. KLF9-S2 was expressed at the highest level among three interference fragments (KLF9-S1, KLF9-S2 and KLF9-S3). KLF9, Kruppel-like factor 9; saRNA, small activating RNA; S1, saRNA 1; S2, saRNA 2; S3, saRNA 3; NC, negative control.

expression may be used as a therapeutic strategy for pancreatic cancer in future (36).

\section{Acknowledgements}

We are grateful to the Molecular Center Laboratory staff of the Second Affiliated Hospital of Nanchang University.

\section{Funding}

The present study was supported from grants from the National Natural Science Foundation of China (no. 81060187) and was partially supported by grants from the Nanchang University Graduate Innovation Foundation (no. cx2016337) of Jiangxi Province, China.

\section{Availability of data and materials}

All data yielded or studied in the present study are included in this published article.

\section{Authors' contributions}

ZZ, FZ, DW, WZ, MW, YZ, JL, LW and XY conceived and designed the experiments. ZZ, FZ, DW, XY and YZ performed the experiments. YZ, JL,WZ, MW and LW analyzed the data. ZZ, FZ, DW, WZ and XY drafted the manuscript. All the authors modified and agreed with the results and conclusions of the study. XY obtained the funding. XY supervised the study. All authors read and approved the manuscript and agree to be accountable for all aspects of the research in ensuring that the accuracy or integrity of any part of the work are appropriately investigated and resolved.

\section{Ethics approval and consent to participate}

The Ethics Committee of the Second Affiliated Hospital of Nanchang University approved our study, and all participants signed written informed consent. The present study was conducted in accordance with the ethical standards of the Helsinki Declaration. 


\section{Patient consent for publication}

Not applicable.

\section{Competing interests}

There authors declare that they have no competing interests.

\section{References}

1. Yang Y, Yan S, Tian H and Bao Y: Macrophage inhibitory cytokine-1 versus carbohydrate antigen 19-9 as a biomarker for diagnosis of pancreatic cancer: A PRISMA-compliant metaanalysis of diagnostic accuracy studies. Medicine (Baltimore) 97: e9994, 2018.

2. Ferlay J, Soerjomataram I, Dikshit R, Eser S, Mathers C, Rebelo M, Parkin DM, Forman D and Bray F: Cancer incidence and mortality worldwide: Sources, methods and major patterns in GLOBOCAN 2012. Int J Cancer 136: E359-E386, 2015.

3. Siegel RL, Miller KD and Jemal A: Cancer Statistics, 2017. CA Cancer J Clin 67: 7-30, 2017

4. German RR, Fink AK, Heron M, Stewart SL, Johnson CJ, Finch JL and Yin D; Accuracy of Cancer Mortality Study Group: The accuracy of cancer mortality statistics based on death certificates in the United States. Cancer Epidemiol 35: 126-131, 2011.

5. Limame R, Op de Beeck K, Lardon F, De Wever O and Pauwels P: Krüppel-like factors in cancer progression: Three fingers on the steering wheel. Oncotarget 5: 29-48, 2014.

6. Heard ME, Simmons CD, Simmen FA and Simmen RC: Krüppel-like factor 9 deficiency in uterine endometrial cells promotes ectopic lesion establishment associated with activated notch and hedgehog signaling in a mouse model of endometriosis. Endocrinology 155: 1532-1546, 2014.

7. Ohguchi H, Tanaka T, Uchida A, Magoori K, Kudo H, Kim I, Daigo K, Sakakibara I, Okamura M, Harigae H, et al: Hepatocyte nuclear factor 4alpha contributes to thyroid hormone homeostasis by cooperatively regulating the type 1 iodothyronine deiodinase gene with GATA4 and Kruppel-like transcription factor 9. Mol Cell Biol 28: 3917-3931, 2008.

8. Bieker JJ: Krüppel-like factors: Three fingers in many pies. J Biol Chem 276: 34355-34358, 2001.

9. Tetreault MP, Yang Y and Katz JP: Krüppel-like factors in cancer. Nat Rev Cancer 13: 701-713, 2013.

10. Yori JL, Johnson E, Zhou G, Jain MK and Keri RA: Kruppel-like factor 4 inhibits epithelial-to-mesenchymal transition through regulation of E-cadherin gene expression. J Biol Chem 285: 16854-16863, 2010.

11. Yori JL, Seachrist DD, Johnson E, Lozada KL, Abdul-Karim FW, Chodosh LA, Schiemann WP and Keri RA: Krüppel-like factor 4 inhibits tumorigenic progression and metastasis in a mouse model of breast cancer. Neoplasia 13: 601-610, 2011.

12. Qiao F, Yao F, Chen L, Lu C, Ni Y, Fang W and Jin H: Krüppel-like factor 9 was down-regulated in esophageal squamous cell carcinoma and negatively regulated beta-catenin/TCF signaling. Mol Carcinog 55: 280-291, 2016.

13. Kang L, Lü B, Xu J, Hu H and Lai M: Downregulation of Krüppel-like factor 9 in human colorectal cancer. Pathol Int 58 : 334-338, 2008

14. Simmen FA, Su Y, Xiao R, Zeng Z and Simmen RC: The Krüppel-like factor 9 (KLF9) network in HEC-1-A endometrial carcinoma cells suggests the carcinogenic potential of dysregulated KLF9 expression. Reprod Biol Endocrinol 6: 41, 2008.

15. Pabona JM, Velarde MC, Zeng Z, Simmen FA and Simmen RC Nuclear receptor co-regulator Krüppel-like factor 9 and prohibitin 2 expression in estrogen-induced epithelial cell proliferation in the mouse uterus. J Endocrinol 200: 63-73, 2009.

16. Pabona JM, Simmen FA, Nikiforov MA, Zhuang D, Shankar K, Velarde MC, Zelenko Z, Giudice LC and Simmen RC: Krüppel-like factor 9 and progesterone receptor coregulation of decidualizing endometrial stromal cells: Implications for the pathogenesis of endometriosis. J Clin Endocrinol Metab 97: E376-E392, 2012.
17. Fu DZ, Cheng Y, He H, Liu HY and Liu YF: The fate of Krüppel-like factor 9-positive hepatic carcinoma cells may be determined by the programmed cell death protein 5 . Int $\mathbf{J}$ Oncol 44: 153-160, 2014.

18. Shen P, Sun J, Xu G, Zhang L, Yang Z, Xia S, Wang Y, Liu Y and Shi G: KLF9, a transcription factor induced in flutamide-caused cell apoptosis, inhibits AKT activation and suppresses tumor growth of prostate cancer cells. Prostate 74: 946-958, 2014.

19. Huang Y, Ju B, Tian J, Liu F, Yu H, Xiao H, Liu X, Liu W, Yao Z and Hao Q: Ovarian cancer stem cell-specific gene expression profiling and targeted drug prescreening. Oncol Rep 31: 1235-1248, 2014

20. Ying M, Tilghman J, Wei Y, Guerrero-Cazares H, QuinonesHinojosa A, Ji H and Laterra J: Kruppel-like factor-9 (KLF9) inhibits glioblastoma stemness through global transcription repression and integrin $\alpha 6$ inhibition. J Biol Chem 289: 32742-32756, 2014

21. Yang M, Ke NW, Zeng L, Zhang Y, Tan CL, Zhang H, Mai G, Tian BL and Liu XB: Survival analyses for patients with surgically resected pancreatic neuroendocrine tumors by World Health Organization 2010 Grading Classifications and American Joint Committee on Cancer 2010 Staging Systems. Medicine (Baltimore) 94: e2156, 2015.

22. Kamarajah SK, Burns WR, Frankel TL, Cho CS and Nathan H: Validation of the American Joint Commission on Cancer (AJCC) 8th Edition Staging System for Patients with Pancreatic Adenocarcinoma: A Surveillance, Epidemiology and End Results (SEER) analysis. Ann Surg Oncol 24: 2023-2030, 2017.

23. Warner SL, Stephens BJ, Nwokenkwo S, Hostetter G, Sugeng A, Hidalgo M, Trent JM, Han H and Von Hoff DD: Validation of TPX2 as a potential therapeutic target in pancreatic cancer cells. Clin Cancer Res 15: 6519-6528, 2009.

24. Frič P, Šedo A, Škrha J, Bušek P, Laclav M, Škrha P and Zavoral M: Early detection of sporadic pancreatic cancer: Time for change. Eur J Gastroenterol Hepatol 29: 885-891, 2017.

25. Chambers AF, Groom AC and MacDonald IC: Dissemination and growth of cancer cells in metastatic sites. Nat Rev Cancer 2: 563-572, 2002

26. Sieber O, Heinimann K and Tomlinson I: Genomic stability and tumorigenesis. Semin Cancer Biol 15: 61-66, 2005.

27. Kang L and Lai MD: BTEB/KLF9 and its transcriptional regulation. Yi Chuan 29: 515-522, 2007 (In Chinese).

28. Lu XJ, Shi Y, Chen JL and Ma S: Krüppel-like factors in hepatocellular carcinoma. Tumour Biol 36: 533-541, 2015.

29. Sun J, Wang B, Liu Y, Zhang L, Ma A, Yang Z, Ji Y and Liu Y: Transcription factor KLF9 suppresses the growth of hepatocellular carcinoma cells in vivo and positively regulates p53 expression. Cancer Lett 355: 25-33, 2014.

30. Limame R, de Beeck KO, Van Laere S, Croes L, De Wilde A, Dirix L, Van Camp G, Peeters M, De Wever O, Lardon F, et al: Expression profiling of migrated and invaded breast cancer cells predicts early metastatic relapse and reveals Krüppel-like factor 9 as a potential suppressor of invasive growth in breast cancer. Oncoscience 1: 69-81, 2013.

31. Tang H, Wei P, Chang P, Li Y, Yan D, Liu C, Hassan M and Li D: Genetic polymorphisms associated with pancreatic cancer survival: A genome-wide association study. Int J Cancer 141: 678-686, 2017.

32. Brown AR, Simmen RC, Raj VR, Van TT, MacLeod SL and Simmen FA: Krüppel-like factor 9 (KLF9) prevents colorectal cancer through inhibition of interferon-related signaling. Carcinogenesis 36: 946-955, 2015.

33. Bogachek MV, De Andrade JP and Weigel RJ: Regulation of epithelial-mesenchymal transition through SUMOylation of transcription factors. Cancer Res 75: 11-15, 2015.

34. Deryugina EI and Quigley JP: Matrix metalloproteinases and tumor metastasis. Cancer Metastasis Rev 25: 9-34, 2006.

35. Kessenbrock K, Plaks V and Werb Z: Matrix metalloproteinases: Regulators of the tumor microenvironment. Cell 141: 52-67, 2010.

36. Ying M, Sang Y, Li Y, Guerrero-Cazares H, QuinonesHinojosa A, Vescovi AL, Eberhart CG, Xia S and Laterra J: Krüppel-like family of transcription factor 9, a differentiationassociated transcription factor, suppresses Notch1 signaling and inhibits glioblastoma-initiating stem cells. Stem Cells 29: 20-31, 2011. 